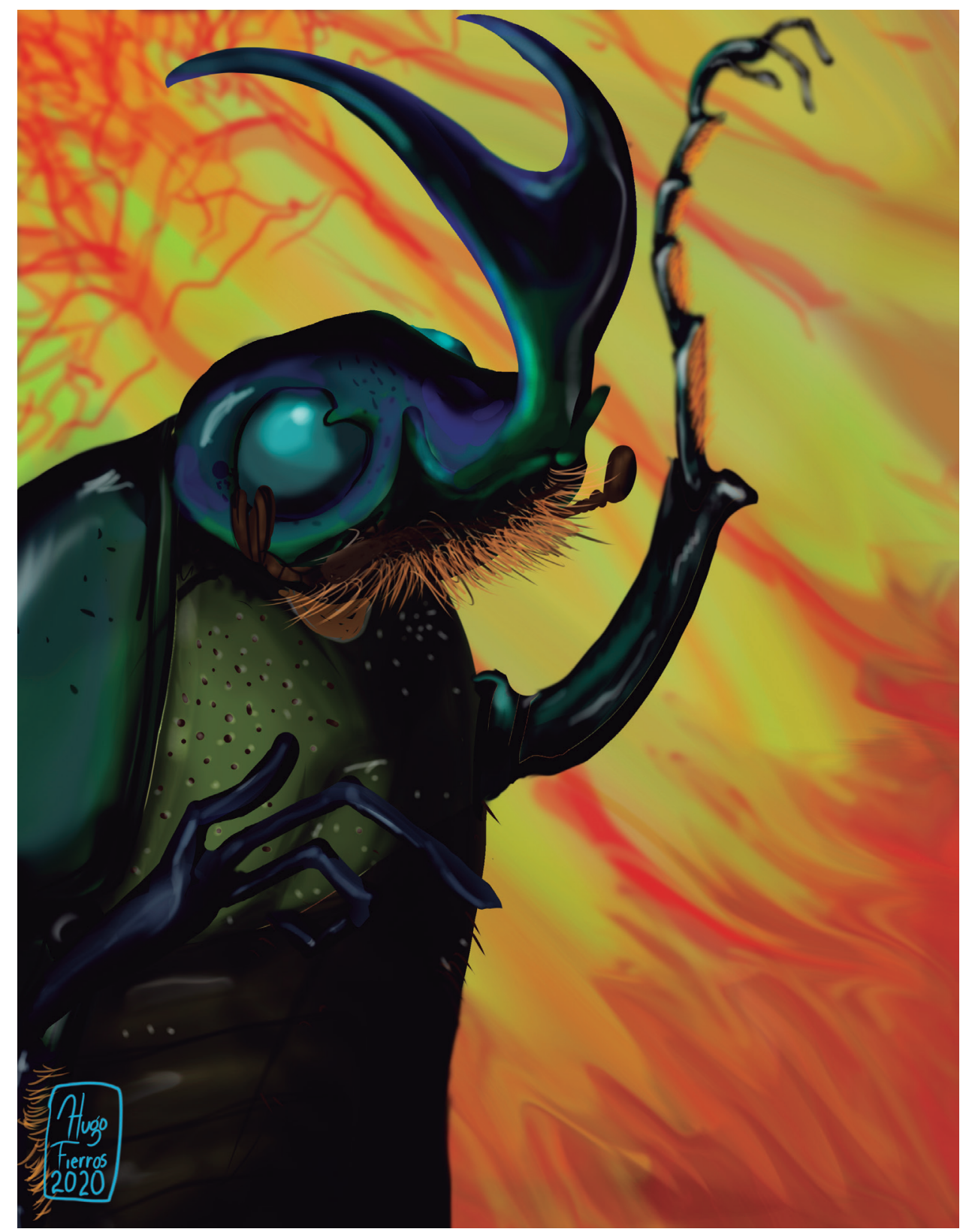

Dugesiana, Año 28, No. 1, (enero-junio, primer semestre de 2021), es una publicación semestral, editada por la Universidad de Guadalajara, a través del Centro de Estudios en Zoología, por el Centro Universitario de Ciencias Biológicas y Agropecuarias. Camino Ramón Padilla Sánchez \# 2100, Nextipac, Zapopan, Jalisco, Tel. 37771150 ext. 33218, http://148.202.248.171/dugesiana/index.php/DUG/index, glenusmx@gmail.com. Editor responsable: José Luis Navarrete-Heredia. Reserva de Derechos al Uso Exclusivo 04-2009-062310115100-203, ISSN: $2007-$ 9133, otorgados por el Instituto Nacional del Derecho de Autor. Responsable de la última actualización de este número: José Luis Navarrete-Heredia, Editor y Ana Laura González-Hernández, Asistente Editorial. Fecha de la última modificación 15 defebrero 2021, con un tiraje de un ejemplar.

Las opiniones expresadas por los autores no necesariamente reflejan la postura del editor de la publicación.

Queda estrictamente prohibida la reproducción total o parcial de los contenidos e imágenes de la publicación sin previa autorización de la Universidad de Guadalajara. 


\title{
In Memoriam: Martín Leonel Zurita García (1979-2020)
}

\author{
Erick Omar Martínez-Luque ${ }^{*}$, María Areyni Zurita-García ${ }^{2}$, Kaedi Zurita García ${ }^{2}$, Santiago \\ Zaragoza-Caballero $^{3 a}$, Paulina Cifuentes-Ruiz ${ }^{3 b}$, Cisteil X. Pérez-Hernández ${ }^{4}$, Sara Lopéz-Pérez ${ }^{5 a}$, \\ Geovanni M. Rodríguez-Mirón ${ }^{5 b}$, Enya Ramírez del Valle ${ }^{6}$, Nayeli Gutiérrez ${ }^{7}$, Erick Antonio Zavala \\ León $^{3 c}$, Daniel E. Domínguez-León ${ }^{3 \mathrm{~d}}$, Mireya González-Ramírez ${ }^{3 \mathrm{e}}$ y Miriam Aquino-Romero ${ }^{8}$
}

${ }^{1}$ Facultad de Ciencias Naturales, Universidad Autónoma de Querétaro, Av. De las Ciencias s/n, Juriquilla, Santa Rosa Jáuregui. C. P.76230. Querétaro, México; ${ }^{2}$ Av. Reforma \#22, Calipan, Coxcatlán, Puebla, México; ${ }^{3}$ Departamento de Zoología, Instituto de Biología, UNAM, Apartado Postal 70-153, 04510, CDMX, México; ${ }^{4}$ Laboratorio de Interacciones Bióticas en Hábitats Alterados, Instituto de Investigaciones en Ecosistemas y Sustentabilidad, UNAM, Antigua carretera a Pátzcuaro \#8701, Col. San José de La Huerta, C. P. 58190, Morelia, Michoacán, México; ${ }^{5}$ Colección Coleopterológica, Museo de Zoología, Facultad de Estudios Superiores Zaragoza, UNAM. Av. Guelatao 66, Ejército de Oriente, Iztapalapa, C. P. 09230, CDMX, México; ${ }^{6}$ Wageningen University and Research, 6708 PB Wegeningen, Países Bajos; ${ }^{7}$ Richard Gilder Graduate School, American Museum of Natural History. Central Park West \& 79th St, New York, NY 10024, USA $;{ }^{8}$ Facultad de Ciencias, UNAM, circuito exterior s/n, C. U., Coyoacán, C. P. 04510, CDMX, México; * erickmtzluque@gmail.com.

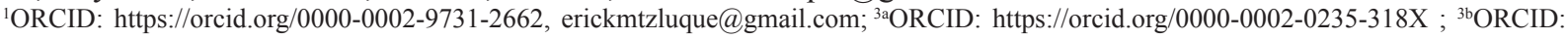
https://orcid.org/0000-0002-0490-7967; ${ }^{4}$ Cisteil X. Pérez-Hernández, ORCID: https://orcid.org/0000-0002-6698-2524 ; ${ }^{5 a}$ ORCID: https://orcid. org/0000-0002-9925-0186; ${ }^{56}$ ORCID: https://orcid.org/0000-0003-0751-4672; ' ${ }^{6}$ ORCID: https://orcid.org/0000-0002-7286-9114; ' ${ }^{7}$ ORCID: https://orcid.org/0000-0002-3057-8167; ${ }^{3 c} \mathrm{ORCID}$ : https://orcid.org/0000-0001-7412-6288; ${ }^{3 d} \mathrm{ORCID:} \mathrm{https://orcid.org/0000-0003-3848-7355;}$ ${ }^{3 e}$ ORCID: https://orcid.org/0000-0002-2277-7991; y ${ }^{8}$ Miriam Aquino-Romero, ORCID: https://orcid.org/0000-0003-1661-3824.

\section{RESUMEN}

A lo largo de este documento se resalta el legado académico y humano de Martín Leonel Zurita-García (1979-2020). Se describe su trayectoria académica sobre todo en su fase universitaria, en la que se dedicó al campo de la Entomología, particularmente de la Sistemática, Biogeografía y Ecología de los coleópteros elatéridos, así como a la Entomología Forense. Publicó 17 artículos científicos y cuatro capítulos de libros; participó en cinco congresos nacionales y dos congresos internacionales. Martín Zurita es autor de siete taxones de Coleoptera. Como docente, impartió las asignaturas de Sistemática, Biogeografía y Entomología Forense, en la Facultad de Ciencias y la Facultad de Medicina de la UNAM, así como en la Universidad Simón Bolívar. Fungió como director o codirector de nueve tesis de Licenciatura en Biología que versan sobre Sistemática, Faunística y Entomología Forense. Asimismo, participó como jurado en cuatro exámenes de grado (tres de Maestría y uno de Doctorado). La segunda parte del documento es testimonial, incluye las sentidas palabras expresadas por hermanos, maestro, amigos, alumnos y colegas en quienes Martín Zurita dejó una profunda huella.

Palabras clave: Coleoptera, coleopterólogo, México.

\begin{abstract}
The academic and human legacy of Martín Leonel Zurita-García (1979-2020) is highlighted in this document. His academic career, especially in his universitarian period, is described. He devoted his research to the field of Entomology, in particular, to the Systematics, Biogeography, and Ecology of elaterid beetles, as well as Forensic Entomology. He published 17 scientific papers, and four book chapters; he also participated in five national and two international meetings. Martín Zurita is the author of seven Coleoptera taxa. He was a teacher of Systematics, Biogeography, and Forensic Entomology subjects at the Sciences School and Medicine School of the National Autonomous University of Mexico (UNAM). He was advisor or co-advisor of nine Bachelor's thesis in Biology, with topics such as Systematics, Faunistics, and Forensic Entomology. He also participated as a jury member in four graduate students' examinations (three Master's degrees and one Ph.D.). The second part of the document is testimonial: it includes the touching words expressed by siblings, advisor, friends, students, and colleagues, in whom Martín Zurita made a deep impression.
\end{abstract}

Key words: Coleoptera, coleoperologist, Mexico. 
Martín Leonel Zurita García nació el 2 de julio de 1979 en el pueblo de Calipan, al sur oriente del estado de Puebla, en el Centro de México. Fue el segundo de cuatro hijos del matrimonio de Romalda García Fernández (1954) y de Ariel Zurita Gómez (1950-1992). Murió el 31 de Julio en Tehuacán, Puebla. Estudió en la Escuela Primaria "Miguel Hidalgo y Costilla" (1985-1991) y en la Escuela Secundaria "Plan de San Luis" (1991-1994), ambas ubicadas en Calipan, Puebla. Asistió a la Escuela Preparatoria Federal "Gilberto Martínez Gutiérrez" de Tehuacán (1994-1997) (Fig. 1).

Desde temprana edad mostró gusto y facilidad por el estudio, particularmente por las matemáticas. Por eso en su casa pensaron que elegiría alguna ingeniería como carrera, pero optó por la biología. Vivió, estudió y trabajó la mayor parte de su vida en la Ciudad de México, donde cursó la Licenciatura en Biología en la Facultad de Ciencias de la Universidad Nacional Autónoma de México (19972001). Su tesis de licenciatura (2002-2004), su maestría (2005-2007) y su doctorado (2008-2012); los llevó a cabo en el Instituto de Biología de la misma universidad, bajo la asesoría del Dr. Santiago Zaragoza Caballero (Fig. 2). Posteriormente fue admitido para una estancia posdoctoral (2013-2014) en la Universidad de Sao Paulo, Brasil.

Como parte de sus diversos proyectos, realizó estancias de investigación en la Estación de Biología de Chamela, Jalisco, México (2001); en el Museum national d' Histoire naturelle, Francia (2005); en el Museo de Historia Natural de Londres, Reino Unido (2005); y en Agriculture and Agri-Food Canadá, Canadá (2010). Su trabajo lo llevó a ser candidato a investigador Nacional del Sistema Nacional de Investigadores (SNI-CONACYT) (2018-2021).

$\mathrm{Su}$ carrera profesional como entomólogo dio inicio en 2006; la cual se enfocó principalmente al estudio de la sistemática, la biogeografía, la evolución y las relaciones ecológicas de los coleópteros de la familia Elateridae en México; esforzándose sobre todo por describir la diversidad biológica de la subtribu Agriotina, así como por expandir el conocimiento de la fauna de elatéridos presentes en México (Fig. 2). Entre sus trabajos, destacan los que realizó sobre los elatéridos presentes en los estados de Morelos, Jalisco, Guerrero y Michoacán.

Martín Zurita escribió 17 artículos científicos, cuatro capítulos de libros (Cuadro 1), y participó en cinco congresos nacionales, dos congresos internacionales y un congreso latinoamericano de entomología. Además, fue autor de los siguientes taxones:

Elateridae (Coleoptera: Insecta)

- Agriotes minutus Zurita-García, 2012

- Agriotes pedregalensis Zurita-García, 2012

- Agriotes rhombus Zurita-García, 2012

- Agriotes trinotatus Zurita-García, 2012

Lampyridae (Coleoptera: Insecta)

- Photinus erici Zaragoza-Caballero y Zurita-García, 2020
- Photinus cempoalli Zaragoza-Caballero y ZuritaGarcía, 2020

- Photinus zempoalensis Zaragoza-Caballero y Zurita-García, 2020

Como docente (2009-2020) impartió cursos de Entomología Forense en la carrera de Biología en la Facultad de Ciencias y Tecnología de la Universidad Simón Bolívar; Biogeografía 1 y Sistemática 1 para la licenciatura de Biología en la Facultad de Ciencias de la Universidad Nacional Autónoma de México; Entomología Forense y Taller de Introducción a la Enseñanza para la Licenciatura en Ciencia Forense de la Facultad de Medicina de la Universidad Nacional Autónoma de México. Impartió también las materias de Sistemática 1 y Sistemática de Insectos para el Posgrado de Ciencias Biológicas en el Instituto de Biología de la Universidad Nacional Autónoma de México.

Estableció una interacción prolífica con colegas de la Licenciatura de Ciencia Forense, así como de la Universidad Autónoma de Morelos, asesorando y co-asesorando alumnos de las instituciones antes mencionadas; dirigió tres tesis de licenciatura, dos en la Facultad de Ciencias, UNAM y una en la Universidad Autónoma Benito Juárez, Oaxaca, México, así como otras seis tesis en las que participaba como asesor y co-asesor (Cuadro 2). Participó en un comité tutoral de maestría (2016-2018) y en uno de doctorado (2019-) del estudiante de doctorado Erick Omar Martínez Luque, ambos en la Universidad Autónoma de Querétaro, México. Además, participó en cuatro exámenes de grado (Cuadro 3) en la Universidad Autónoma de Morelos y la Universidad Nacional Autónoma de México.

Preocupado por la conservación de la biodiversidad de nuestro país, formó parte del proyecto de investigación "Estudio para la expedición de declaratoria como Área Natural Protegida del Cerro del Palenque", en el municipio de Purísima del Rincón, en el Estado de Guanajuato, México; colaboró en 12 proyectos de divulgación y difusión científica en la Universidad Nacional Autónoma de México y un proyecto de divulgación científica en la Universidad Autónoma de Zacatecas, México. Asimismo, trabajó de 2007 a 2008 en el Instituto Nacional de Ecología (ahora INECC) en la evaluación de riesgo de cultivos transgénicos. Martín Zurita fue un gran científico que, a pesar de su pronta partida, fue un pilar en la formación de varias generaciones de entomólogos y coleopterólogos mexicanos, caracterizándose por su disposición, amabilidad, sencillez y sobre todo por su entusiasmo y amor por los insectos (Fig. $3)$. En su pueblo natal, Martín motivó y fue un ejemplo para que sus hermanos y sobrinos continuaran estudiando y buscaran también oportunidades de formación académica en la Ciudad de México.

\section{María Areyni Zurita García}

Querido hermanito...

¿Cómo recordarte?... Creo que sería en los momentos 
felices, en el tiempo que compartimos, con los consejos, con los mensajes, con tantas pláticas... con lo plena que fue mi vida junto a ti.

Es inevitable sentir tristeza, dolor y hasta egoísmo... ¿Por qué tú?, no lo comprendo...

Nunca estuve preparada para tu partida. Todos dicen "con el tiempo", todos dicen muchas cosas; yo sólo sé que te llevaste una parte de mi vida. Siempre me harás falta. Cada día intento pensar que estas en uno de tus tantos viajes y que pronto volveremos a encontrarnos. Sé que por ahora te seguiré llorando hasta agotar mi última lágrima.

No tengo idea de cómo fue que guardé uno de tus correos y hasta lo imprimí, pero comparto tus palabras hermanito: "Tú haz lo que creas correcto. Que de algo te sirva que hayas dado ese tiempo a la educación inicial. Bueno hermanita, tú tranquila y calmada, trata siempre de ser feliz, que tienes a tus hermanos para cualquier cosa, por algo somos tres."

Serían muchas palabras, muchos escritos para hablar sobre tu persona, pero como hermano fuiste único y si me dieran la opción de decidir, te volvería a elegir como mi hermanito. Cuanta falta me haces mi negris, así te llamaba, no sé cuándo, pero volveremos a encontrarnos mi querido Dr. Martín Leonel Zurita García.

\section{Kaedi Zurita García}

A mi hermano y amigo, que siempre estuvo incondicionalmente a mi lado, en los buenos y malos momentos de mi vida. Siempre estaré agradecido por sus palabras, consejos, y esos regaños por ser un mejor estudiante, pero sobre todo por su calor de ser un magnifico hermano.

Esas vivencias juntos, de jugar futbol, de preguntarme siempre como iba yo en la escuela, de revisarme mis tareas y hacerme preguntas de lo aprendido en clase, de sentarme a su lado y explicarme cosas que no entendía de la materia, de ir al cine, de ir de compras y esas visitas al campo de las cuales aprendí el amor que le tenía mi hermano a su profesión, pero en particular de sus enseñanzas como un gran hermano y una excepcional persona, que siempre se preocupó por mi familia y por mí, quedaran plasmadas en este corazón que lo seguirá amando y recordando por el resto de mi vida.

A mi hermano que con su humildad y conocimiento de lo que le gustaba aprender, llego hasta lo más alto en su desarrollo de su profesión, me sentiré por el resto de mis días orgulloso y honrado de todos sus triunfos y logros como el gran profesionista que fue.

Algún día nos volveremos a ver hermano, hasta ese momento siempre sabrás, que nunca dejaste de ser el mejor hijo, hermano, amigo y sobre todo un gran ser humano. Siempre estarás presente en nuestros corazones mi querido y amado hermanito. Hasta el cielo te mando miles de besos y un fuerte abrazo con todo mi amor de tu hermano Kaedi que nunca se olvidara de ti Dr. Martín Leonel Zurita García. "Quiero romperme en millones de pedazos en el tiempo espacio, para que uno de ellos tenga la oportunidad de encontrarte y que puedas sonreírle" Marleo.

\section{Santiago Zaragoza Caballero}

Siempre es triste despedirse de una persona con la que se ha convivido muchos años. Martín fue discípulo, colega y amigo. Poco después de iniciado el milenio, Martín llegó procedente de la Facultad de Ciencias de la Universidad Nacional Autónoma de México, mostrando interés por el estudio de los escarabajos y particularmente de los elatéridos, o escarabajos click.

Invité a Martín a estudiar la familia Elateridae en la Reserva Sierra de Huautla como parte de un proyecto ya en curso desde octubre de 1995 "Los insectos asociados al bosque seco", particularmente de la vertiente del Pacífico Mexicano. El proyecto se desarrolló con un método que implicó trabajo de campo por siete días de cada mes a lo largo de siete años. En las recolectas se recuperaron representantes de varios órdenes de insectos y de varias familias de escarabajos. El estudio que Martín llevó a cabo fue su tema de tesis de licenciatura, mismo que defendió exitosamente en septiembre de 2004.

Nuestra relación se hizo continua cuando Martín inició su trabajo en la "Sistemática de la subtribu Agriotina (Coleoptera: Elateridae: Elaterinae Agriotini)" como proyecto de sus estudios de Maestría, que implicaron la organización de los ejemplares perteneciente a esa tribu, depositados en la Colección Nacional de Insectos del Instituto de Biología de la Universidad Nacional Autónoma de México. Posteriormente, el conocimiento adquirido y su inquietud de conocer más profundamente a los elatéridos, lo llevó a desarrollar como tema doctoral la "Revisión taxonómica y análisis filogenético del género Agriotes en el Continente Americano".

$\mathrm{Su}$ preparación académica en general se consolidó con sus estudios posdoctorales y estancias en diversas instituciones tanto del país como del extranjero, esto le permitió participar como autor o coautor de varios artículos publicados sobre diversos temas. Así, en orden cronológico, fueron elaborándose trabajos como: "Patrones de distribución de la familia Lycidae (Coleoptera)", trabajo que nos permitió en 2006, ofrecer una visión panorámica de la distribución de las especies de los insectos con los élitros articulados en el territorio nacional. El texto * "La familia Elateridae de la Reserva de la Biosfera Sierra de Huautla, Morelos, México" de 2007, que recoge información tanto taxonómica como de la distribución temporal de los escarabajos que impelen, entre los que está Pyrophorus mexicanus, que los aztecas conocían como "el que tiene lumbre". El artículo *"Elateroidea según Crowson ¿Un grupo monofilético?" de 2010, responde a una cuestión heredada, concluyéndose que se trata de un grupo parafilético. La revisión del género Agriotes, integrado por 28 especies implicó la publicación en 2012, de Agriotes minutus, A. pedregalensis, A. rhombus, and A. trinotatus. Se describen nuevas especies en el artículo 
* "New species of Agriotes (Coleoptera: Elateridae) from Mexico with a key to species". En * "Biodiversidad de elatéridos (Coleoptera) en México" de 2014, sintetiza toda la información de la familia en nuestro país. Finalmente, en "A preliminary study on the phylogeny of the family Phengodidae (Insecta: Coleoptera)" de 2015, proponemos el primer estudio filogenético de esa familia de escarabajos. Como director de tesis, Martín propone el estudio de la "Distribución temporal de los insectos del suelo en el bosque tropical caducifolio de Santiago Dominguillo, Oaxaca" 2016. Dominguillo, Oaxaca que fue otra localidad estudiada en nuestro proyecto, como lo es también Ixtlahuacán, Colima de donde se analiza la abundancia, riqueza y estacionalidad de las familias Cantharidae, Lampyridae, Lycidae, Phengodidae y Telegeusidae en el capítulo de 2016 "Escarabajos de Ixtlahuacán (Elateroidea)". En "A checklist of the Coleoptera (Insecta) from Morelos, Mexico" de 2019, se agregaron más de mil especies a la fauna conocida de escarabajos para Morelos y en 2020 publicamos los artículos "Luciérnagas del centro de México (Coleoptera: Lampyridae): descripción de 37 especies nuevas"; *“Las avispas Vespa Linnaeus (Vespidae: Vespinae) y su Goliat la Vespa mandarinia Smith; "Luciérnagas de México". Los cuatro últimos trabajos fueron elaborados con la participación del grupo que se fue integrando a través de los años en primer lugar: Martín L. Zurita-García, seguidos de Paulina Cifuentes-Ruíz, Cisteil X. Pérez-Hernández, Mireya González-Ramírez, Sara López-Pérez, Viridiana Vega-Badillo, Daniel L. Domínguez-León, Geovanni M. Rodríguez-Mirón, Ishwari G. Gutiérrez-Carranza, Enya Ramírez del Valle y Nayeli Gutiérrez, que, afortunadamente llegaron a realizar a nuestro laboratorio sus proyectos de tesis para después convertirse en, colegas, compañeros y siempre amigos; y quienes en los últimos años han sido mi fortaleza.

Ellos, me acompañaron a la inauguración del "Museo de las Luciérnagas" donde se develó una placa alusiva en la Ex Hacienda Ixtafiayuca de Nanacamilpa, Tlaxcala, portando con orgullo una playera con la imagen impresa de Photinus congruus Gorham.

Me hicieron fuerte en 2012, en la instalación de 18 trampas de interceptación en Chamela, Jalisco, cuyo objetivo es registrar la riqueza y abundancia tanto vertical como estacional de la fauna de insectos. Actualmente, siguen en operación. Martín, particularmente me acompañó a la instalación de ese sistema de trampeo como fase experimental en el Jardín Botánico del Instituto de Biología. El grupo también me ha apoyado en algunas modificaciones que hemos hecho con diferentes tipos de trampas.

Hago estos comentarios, tratando de destacar la cohesión del grupo; en realidad no podría decir de quien fue la idea integrativa, lo que sí puedo comentar es el comportamiento crítico por demás agudo de Martín o cualquier otro miembro, para recomendar cambios en sus presentaciones o escritos, antes de ponerlas a mi consideración.

Otra idea del grupo fue manifestarse como integrantes del Laboratorio Zaragoza con un logo alusivo, impreso en sus playeras.

A título personal, Martín subió a la red proyectos que nos comprometían a seguir trabajando en grupo.

\section{Paulina Cifuentes Ruiz}

A lo largo de 18 años, coincidí con Martín no solo en el laboratorio del Dr. Santiago Zaragoza Caballero, también en exámenes de grado, salidas a campo, festejos, salidas con amigos y hasta bodas. Cuando acudí por primera vez al cubículo del Dr. Zaragoza fue para la determinación taxonómica de un fósil, a principios del 2002. Martín realizaba entonces su tesis de licenciatura y estaba discutiendo con el Dr. Zaragoza un término entomológico: "fóvea". Me saludó con una sonrisa, siempre fue cálido con quien acababa de conocer y por supuesto, con quien ya conocía. Llevaba una camiseta con un estampado de una pirámide y de glifos, considero este detalle significativo porque Martín siempre estuvo orgulloso de nuestra raíz indígena. Regresé al cubículo en el 2003, en donde permanecí para hacer mi servicio social y mis tesis. A lo largo de ese tiempo, siempre recibí de Martín sabios y atinados consejos, ayuda técnica, escucha y compañía; en suma, amistad y tutoría sincera. Martín fue un compañero muy talentoso y entrañable, siempre incluyente en los proyectos que se desarrollaban en el laboratorio y guiando de buena fe a quien acudía con él. Fui testigo de cómo pasó de ser discípulo del Dr. Zaragoza a ser su colega, su par. Siempre fue muy independiente, y su talento no se limitó a la entomología. No pocas veces lo encontré regresando de sus paseos sabatinos por el Jardín Botánico y sus alrededores tomando fotografías de gran calidad de la naturaleza que ahí habita. Martín tuvo la capacidad de ampliar sus horizontes académicos involucrándose en proyectos de entomología forense. Recuerdo sus inicios en ese campo, dando clases en la carrera de Ciencias Forenses de la Facultad de Medicina de la UNAM y cómo poco a poco fue conduciendo más trabajos de tesis al respecto. Siempre fue un hombre generoso con su conocimiento, un par de veces le pedí que acudiera a dar pláticas de entomología forense a alumnos de nivel preparatoria y siempre aceptó de buena gana.

No podemos honrar la memoria de la gente valiosa que parte repentinamente tratando de emular su talento, pues eso es un sello personal. Podemos, sin embargo, recordar la generosidad o la buena actitud de estas personas e intentar imprimir algo de eso en nuestras acciones cotidianas. Hay una frase que Martín incluyó en la dedicatoria personal de dos de sus tesis: "Al juego entre la vida y la muerte". La vida y la muerte coexisten, siempre tendremos que resignificar nuestra vida ante la muerte de las personas que queremos. Gracias por todo Martín.

\section{Cisteil X. Pérez Hernández}

En octubre de 2006, por recomendación de Perla Cuevas, conocí a Martín Zurita. Él me recibió con su típica 
amplia sonrisa y con todo el entusiasmo de quien disfruta en exceso lo que hace. Martín me explicó cómo era la dinámica en el cubículo del doctor Santiago y lo mucho que podría aprender si trabajaba ese laboratorio. Me fui a casa contenta y regresé al día siguiente para quedarme en la Colección Nacional de Insectos, por casi 12 años, trabajando con el Dr. Santiago Zaragoza. El recibimiento de Martín no fue fortuito, pues era él quien siempre daba la bienvenida a los nuevos integrantes del laboratorio de Coleoptera. Esa era una de sus principales tareas autoasignadas $\mathrm{y}$, en gran medida, era un detalle que te motivaba a quedarte.

Durante mi paso por ese laboratorio, algunas de las cosas que más disfruté fueron el apoyo, amistad y gran compañerismo de Martín. Con él aprendí muchas cuestiones básicas de la taxonomía y faunística, así como buenas prácticas en la entomología y en las colecciones científicas. Los primeros años solía quedarse conmigo trabajando en el laboratorio hasta las 8 o 9 de la noche, y después me acompañaba hasta el metro Ciudad Universitaria. Martín era un excelente compañero que compartía su conocimiento y experiencia sin esperar nada a cambio. Te hacía sentir que el laboratorio era un lugar vasto y abierto. Era una buena compañía en las largas horas al microscopio. Era una persona sencilla y siempre podías contar con él para lo que se presentara.

A Martín lo conocí primero como un compañero que te contagiaba su experiencia, y más adelante también lo vi como un colega con quien podías discutir los temas que más te apasionaban. Con él se podía hablar de taxonomía, sistemática e insectos -que fueron siempre temas que le entusiasmaban demasiado-, lo mismo que platicar sobre libros, películas, series de TV, fotografía, música y, por supuesto, de café y mezcal.

Durante los últimos años, Martín y yo tomamos caminos académicos distintos. Quizá gracias a eso pude notar cómo fue creciendo cada vez más como profesor de la Facultad de Ciencias y de la Facultad de Medicina de la UNAM, en donde era muy querido y admirado por ser un buen maestro. También vi cómo su experiencia lo llevó a titular a distintos alumnos y alumnas. Además, varios investigadores del Instituto de Biología acudían a él constantemente para que los auxiliara con distintos asuntos. Martín era siempre muy solicitado y él siempre brindaba todo el apoyo que pudiera dar. El querido compañero y amigo dedicó mucho tiempo de su vida a la Colección Nacional de Insectos, a la academia, a la entomología, a sus amistades y colegas. Como tantos de nosotros, él esperaba cualquier oportunidad para tener un lugar propio en donde pudiera seguir cultivando su pasión por la ciencia. Pero es difícil tener esas oportunidades cuando no hay muchas puertas abiertas.

Su temprana partida nos tomó por sorpresa a todos y aún en estos momentos resulta difícil aceptar su pérdida. Hemos perdido un pedacito de ese espíritu entomológico que nos impulsaba a seguir amando la ciencia y los insectos. Sin embargo, tenemos muchos buenos recuerdos de Martín y nos queda la experiencia compartida. No olvidaremos nunca su paso por este mundo y las marcas que dejó en cada uno de nosotros seguirán siempre presentes. Martín, querido amigo y compañero, a donde quiera que vayamos tomaremos un café, un mezcal y una fotografía en tu memoria, hasta que sea el universo quien nos vuelva a juntar en un pedacito de ala de insecto, en la tinta de algún libro, en el brillo de los cocuyos. ¡Hasta siempre, Martincillo!

\section{Sara López Pérez y Geovanni M. Rodríguez Mirón}

No pasó mucho tiempo después de conocerlo por primera vez para que Martín se convirtiera en un personaje principal en nuestras vidas. Cada día de ordinario aparecía en alguna o todas las escenas. Martín fue en principio nuestro maestro, y cada día que pasaba era imposible no llegar a ser amigos, la sangre nos llamaba. Fue ese amigo que siempre estaba ahí para cualquier cosa, siempre con tiempo para escuchar y darte un buen consejo. Siempre disponible para discutir sobre cualquier cosa, aunque en ocasiones discrepábamos en nuestras ideas, nunca hubo rencores, siempre respetó las ideas contrarias. Así como podía ser el Dr. Zurita, en una noche de diversión pasaba a ser esa persona con la que reíamos hasta que amaneciera. Fueron muchas las tardes y noches de relajación, celebración o de cualquier pretexto de diversión, ya fuera con una simple taza de café y galletas de animalitos, con pizza y cerveza o con un buen mezcal. Son muchas las anécdotas de todos juntos riéndonos de la vida y alguna que otra vez filosofando sobre la amistad y otras cosas banales. A Martín nunca le faltaron amigos, su humildad y amabilidad hacía que cualquier persona se sintiera en confianza con él. Fue una persona que siempre se alegraba de los éxitos de sus amigos, al grado de presumirlos. Siempre estaba dispuesto ayudar a las personas, ya fueran alumnos o investigadores, en tareas básicas o para discutir asuntos de proyectos académicos.

A pesar de nuestra amistad él fue siempre una persona reservada, pocos lograron que abriera su corazón, poco supimos de él... Te vamos a extrañar.

\section{Erick Omar Martínez Luque}

A lo largo de nuestras vidas, conocemos a muchas personas con las cuales formamos lazos, creamos vínculos, y forjamos nuestra historia. Sin embargo, muy pocas personas influyen en nuestras vidas tanto que pueden cambiar su rumbo... Me atrevo a decir que Martín Zurita fue una de esas personas, que influyó en gran manera en la persona que soy ahora. No solo porque me instruyó y me compartió su conocimiento sobre el grandioso mundo de los elatéridos a lo largo de más de 11 años si no, porque cada una de sus pláticas y consejos, siempre fueron el sostén que me impulsaba para enfrentar los grandes cambios y a tomar las mejores decisiones que dieron y dan rumbo a mi vida profesional y personal.

Agradezco haber conocido a Martín, quien fue mi maestro y mi colega. Con quien compartí y disfruté tardes e incluso noches y fines de semana analizando y determinando elatéridos al ritmo del reggae y con el buen aroma y sabor 
de una taza de café. De igual forma, estoy agradecido con Martín, porque siempre estuvo a mi lado desde la elaboración de mi tesis de licenciatura, durante el proceso de mi maestría y en esta primera parte de mi doctorado, en la cual formó parte de mis comités tutorales, asesorándome en los diferentes procesos, aportando siempre buenas ideas y comentarios acertados, así como apoyándome en todos los procesos a pesar de la distancia, la cual nunca fue impedimento, ya que siempre mantuvimos una buena relación y comunicación, que nos ayudó a continuar con algunos proyectos.

Su repentina partida nos sorprende a muchos, ya que los que tuvieron la fortuna de conocer a Martín sabían que era una persona alegre, sana, deportista, llena de proyectos, y sobre todo de amigos. Siempre te recordaré, mi estimado amigo, cada vez que vea un Agriotes, cada vez que me encuentre en campo y vea brillar a un cocuyo, cada que esté frente al microscopio analizando elatéridos, siempre recordaré tus consejos y enseñanzas... Hasta siempre mi estimado Keréto.

\section{Enya Ramírez del Valle}

Conocí al Dr. Martín Zurita en el laboratorio de Coleoptera del Dr. Santiago Zaragoza en el 2013. Recuerdo perfectamente que estaba sentado leyendo un libro de sistemática, nos saludamos y platicamos sobre lo que nos interesaba, los insectos. Durante el tiempo que estuve realizando mis estudios de licenciatura en el laboratorio, Martín fue un profesor que amaba su profesión, tenía mucha paciencia para explicar la taxonomía y sistemática de los insectos, y siempre ayudaba cuando un alumno lo necesitaba. Recuerdo cuando trabajé con él revisando unos ejemplares de elatéridos y era de notarse lo mucho que le fascinaba enseñar. Durante ese tiempo además de apender mucho, conocí su buen gusto musical, por ejemplo la banda Joy Division, y la canción que nos gustaba en ese entonces era Disorder.

Estoy muy agradecida con Martín porque fue quien me impulsó a combinar mi pasión por el arte y la ciencia al realizar mi tesis de licenciatura en ilustración científica aplicada a la entomología. Gracias a él me supervisó la Dra. Paulina Cifuentes y el Maestro de Artes Aldi de Oyarzabal, ambos profesionales y excelentes mentores. Aunque Martín no fue mi supervisor oficial, para mí fue como uno, ya que estuvo presente enseñándome y revisando mi trabajo de tesis. Considero que Martín inspiró a muchos estudiantes y dejo valiosas enseñanzas en cada uno de nosotros. Agradezco infinitamente al Dr. Martín por todo su apoyo.

\section{Nayeli Gutiérrez}

No puedo pensar en el laboratorio del Dr. Zaragoza sin Martín quien, como buen foráneo, me recibió con mucha calidez cuando llegué a la CDMX a empezar mi maestría. Recuerdo que me ayudó a familiarizarme con el funcionamiento del laboratorio y de la vida en el Instituto de Biología en general. Ésa es la primera cosa que le agradezco.
Después compartimos el ímpetu para trabajar los fines de semana, en los que poníamos música muy variada. Él tenía un gran gusto por la música que se reflejaba en su avidez por conocer nuevas propuestas. Generalmente salíamos juntos del instituto y caminábamos hasta la entrada de mi edificio en Copilco, el cual quedaba cerca de la parada del transporte que lo dejaba en su casa. Recuerdo con mucho cariño esas caminatas en las que pasábamos por el campus central de CU, y en el proceso, hacíamos la recapitulación de lo que había pasado en el día, a la par que compartíamos impresiones sobre nuestras vidas acompañadas por su ánimo jovial y alegre. A veces, junto con Ishwari Gutiérrez, íbamos a cenar garnachas; fue gracias a Martin que me animé a combatir mi aversión a la carne y probar las quesadillas de picadillo, sus favoritas. Por supuesto le agradezco también su apoyo como mentor durante todo mi proceso de maestría. Martín era muy generoso con su tiempo y no dudaba en interrumpir momentáneamente sus actividades para ayudar a quien solicitara su consejo. No era raro que se tomara un momento para ver en el microscopio los escarabajos que yo estaba estudiando y que juntos discutiéramos mis dudas. Su gusto por los insectos jamás se limitó al grupo que él estudiaba, ya que tenía una gran capacidad de asombro y apreciación por otros grupos. Otra característica que resaltaba de su personalidad era su capacidad de empatizar con gente muy diversa y forjar amistades con personas de diferentes edades e intereses. Su amistad se reflejaba en muchos aspectos, uno de ellos es que impulsaba a sus amigos a cumplir sus metas. Por ejemplo, él me ayudó a iniciar la conversación con el Dr. Santiago cuando yo estaba interesada en escribir un texto sobre la historia de la colección de insectos, y revisó todas las versiones hasta que el texto estuvo terminado. También fue Martín quien me motivó a elegir una introducción más cursi y novelesca en mi ensayo de admisión al doctorado, algo con lo que yo me debatía en el momento. Recuerdo que me dijo que le gustaba más que 'mis otras opciones frías y concisas', y que estuvo a mi lado en el momento en el que di el click en "enviar". Recordaré a Martín con la canción de Víctor Jara "te recuerdo Amanda", que a ambos nos gustaba, y me quedo con la confianza de que su legado de amistad y amor por los insectos estará presente en todos los que tuvimos la suerte de caminar la vida junto a él.

\section{Erick Antonio Zavala León}

Mi primer encuentro con el Dr. Martín Zurita fue en un lejano verano del 2014, en aquel entonces yo contaba con las típicas preocupaciones de un estudiante de licenciatura tales como liberar el servicio social, conseguir un asesor de tesis, concluir trabajos escolares, entre otros; pero nunca imaginé el impacto que representaría en mi vida conocer al Dr. Zurita.

En aquel entonces mis intereses dentro de la biología se enfocaban en la mastozoología, particularmente en el orden Chiroptera y en especial sobre aquellas especies que se alimentaban de insectos. Llegué al laboratorio 
del Dr. Santiago Zaragoza para poder realizar ahí mi servicio social con la intención de aprender taxonomía de insectos y comenzar una tesis en la descripción de dietas de murciélagos insectívoros, pero por azares del destino ese trabajo nunca se realizó y terminé haciendo una tesis en distribución temporal de insectos epigeos de Santiago Dominguillo, Oaxaca, bajo la dirección del Dr. Zurita.

Debo mencionar que la elaboración de ese trabajo de tesis fue de las experiencias más enriquecedoras de mi vida, ya que Martín no se conformó con solo limitarse a enseñarme cuestiones académicas, la formación que me dio fue más allá que solo identificar insectos, sino que me ayudó a mejorar como persona desarrollando un sentido crítico y viendo el lado positivo en todo tipo de situaciones. Recuerdo que nuestras platicas iban desde un análisis de resultados y podían terminar en el análisis de un partido de futbol del fin de semana o compartiendo anécdotas de salidas al campo. Martín siempre fue un ejemplo para todos los integrantes del Laboratorio del Dr. Zaragoza, fue una persona dedicada a su trabajo y a sus alumnos; siempre vio la forma en que los estudiantes adquirieran más que simple información, pues a él le preocupaba que tuvieran un sentido crítico y que su formación fuera más holística. Recuerdo bien la ocasión en que me animó a que participara en el Congreso Nacional de Entomología en Querétaro presentando los avances que teníamos hasta ese entonces de la tesis.

Preparar la ponencia para ese congreso fue un proceso bastante complicado, era mi primera participación en este tipo de eventos y no me sentía preparado, pero Martín siempre estuvo ahí orientándome para estar listo. Cuando llegó la semana del evento, pude notar que Martín estaba feliz de estar ahí, de saludar a sus viejos colegas y conocidos, de escuchar ponencias o leer carteles de su interés, ahí me di cuenta de que a él de verdad le apasionaba su profesión. En lo personal, la participación en el congreso fue buena y tuve una gran retroalimentación de otros especialistas cuyos comentarios me ayudaron a seguir mejorando mi trabajo de tesis. Entonces entendí la razón por la que Martín quería que participara en ese congreso.

Así, después de la experiencia del congreso, terminar el trabajo de tesis y los trámites burocráticos en la facultad; llegó el tan ansiado día para Martín y para mí, el día del examen profesional. Para mí era importante porque significaba terminar con mi formación académica y para Martín era importante porque era el día que el primer alumno al que dirigía como asesor se recibía como profesionista. Cuando concluyó la presentación del trabajo de tesis, Martín fue el primero en iniciar con la ronda de preguntas, una de las cuales fue: "¿Recomendarías el grupo de los insectos a los nuevos estudiantes?". Fue la pregunta más extraña de toda la sesión y que no supe cómo responder.

Hoy con tu pronto partir, Martín, me doy cuenta que con esa pregunta lo que intentabas decirme era que continuara con tu legado, que fuera a lo que me dedicara una vez que abandonará los salones de clases, y que lo hiciera con la misma pasión y dedicación con la que tu hacías tu trabajo como docente, que le enseñara a todos los que pudiera y que nunca fuera egoísta en compartir mi conocimiento. Todos estos momentos compartidos fueron muy enriquecedores a tu lado, porque no solo fuiste un maestro que terminó de formar a un profesionista, fuiste un amigo que me enseñó valores y ayudaste en mi crecimiento como persona.

Todos los que tuvimos la dicha de ser tus alumnos te recordaremos con mucho cariño y siempre te estaremos agradecidos por compartir tu conocimiento con nosotros, la última tarea que nos dejas es seguir inspirando a las nuevas generaciones, difundir todo lo que nos enseñaste no solo dentro de un aula o laboratorio sino también como persona, enorgullecerte con nuestros logros y seguir aprendiendo para continuar tu legado. Gracias por todo Martín Zurita.

\section{Daniel E. Domínguez León}

En los últimos años, sin duda alguna, con quien más aprendí a disfrutar de la entomología fue con mi amigo Martín. Recuerdo aquella ocasión en la que emprendimos el alocado proyecto del ciclo de vida de una luciérnaga del Pedregal de San Ángel. En el mes de julio, durante las noches, bajábamos caminando del Instituto de Biología dispuestos a la colecta de estos peculiares animalitos. Dado su comportamiento teníamos que esperar a que oscureciera, y en el momento en el que desaparecía toda pista de luz solar nos disponíamos a la búsqueda sobre todo de las hembras de esta especie. Martín hacía lucir aquella habilidad que dan los años de experiencia capturando una cantidad increíble de especímenes, y no solo eso, sino que compartía conmigo cada pizca de conocimiento; después de estar algunas horas bajo la lluvia y quedar la mayoría de las veces empapados siempre nos dirigíamos a celebrar ya fuera con unos tacos, crepas, hamburguesas o en cualquier puestecito de comida que se nos cruzara en el camino hacia nuestros hogares. Ya era una costumbre el trabajar hasta tarde, pero hubo una ocasión en particular, cuando todo el equipo decidimos irnos temprano y dejar la entomología por un rato; justo cuando cerrábamos el laboratorio Martín dijo con su clásica sonrisa: "Híjole ¿qué creen?... ¡ están naciendo las peques!”. Emocionado, Martín prendió la computadora y la cámara, y enfocó una caja Petri con un montón de huevos de luciérnagas; al ponerlas en el monitor de inmediato nos dimos cuenta de que las larvas estaban abriendo con sus mandíbulas aquella pequeña prisión que eran sus huevos. Martín no dejaba de grabar, hacer algunas notas, mediciones y separar estos pequeños ejemplares, tanta fue la emoción que no nos dimos cuenta de que ya habían pasado tres horas y que los tres integrantes del equipo (Martín, Ishwari y yo) teníamos un sinnúmero de llamadas y mensajes de nuestros amigos y familiares cercanos. Aquel día los tres nos iríamos felices, ya que era el primer pasito de un gran proyecto; Martín, como de costumbre, se había ofrecido a venir el fin de semana a continuar trabajando con las larvas. Ese día quedé anonadado, pues nunca vi a un entomólogo amar con tanto cariño lo que hace, enseñar a otros y sobre todo hacer que otros sientan esa pasión y se dispongan a seguir este incierto camino. 


\section{Mireya González Ramírez}

Conocí a Martín durante 2013, cuando acudí al laboratorio del Dr. Santiago Zaragoza para realizar mi servicio social y tesis de licenciatura. Durante este tiempo compartimos pláticas y salidas al campo a la Estación de Biología en Chamela, este período me permitió conocerlo y descubrir la gran calidez humana que siempre nos brindó. Martín siempre disfrutó salir al campo y compartir con amigos o alumnos todos los conocimientos que pudieran ayudarte a obtener excelentes resultados. En mi caso, no solo me ayudó en el desarrollo de mi tesis de licenciatura, sino que también me brindó su ayuda y consejo para poder entrar al posgrado, me acompañó a mi entrevista y hasta el último momento me ofreció sus sugerencias para evitar estar nerviosa. Esto se repitió con cada integrante del laboratorio del Dr. Zaragoza, porque siempre estuvo dispuesto a regalarnos un pedacito de su experiencia adquirida a lo largo de estos años. Esa seguridad que lo caracterizaba y que podía contagiarte, es la misma que le permitió entablar amistad con un sinnúmero de personas, los cuales pueden ser testigos de la sencillez y amabilidad que nos regaló como amigo. Para muchos de nosotros Martín fue maestro, amigo, cómplice y compañero de viaje. Es grato recordar las innumerables reuniones que compartimos en casa de amigos, cumpleaños, comidas de fines de año, salidas al campo, congresos, hobbies, pláticas y tazas de café (las cuales nunca rechazaba). Es difícil imaginar que no podremos seguir disfrutando de una pequeña caminata por el Jardín Botánico para compartir nuestro gusto por los cactus y suculentas; sin embargo, estoy segura de que volveremos a coincidir en algún momento. Gracias por tanto Martín.

\section{Miriam Aquino Romero}

Conocí a Martín a inicios de la carrera, hace unos tres años. Él era profesor de Biogeografía de mi gran amigo Andrés Garibay, y ofreció un espacio para hacer un servicio social en la CNIN. Andrés me comentó y sin dudarlo contacté al Dr. Zurita, siempre fue mi sueño trabajar con escarabajos.

Martin me brindó la oportunidad de hacer el servicio social con él, y después de una increíble salida a campo por el proyecto de tesis de mi hermana, Mónica Espadin, también fue mi tutor de tesis de licenciatura con un proyecto de revisión taxonómica de la subfamilia Languriinae, uno de sus grupos hobby.

A veces no sabes que una persona te marcará tanto cuando la conoces, no solemos pensar que perderemos a las personas que tanto queremos. Martín fue de esas personas que imaginas que estarán contigo siempre. Lo conocí para hacer un servicio social y terminó enseñándome no solo sobre escarabajos, él fue mi maestro de vida. Es esa persona en la que me quiero convertir algún día, aunque sea un poco. Me enseñó a cazar bichos y también un Pokémon; a defender mis trabajos y mis sueños; a disfrutar de las noches frías en campo y el café cargado por las mañanas.

Jamás podré agradecerle por ser el mejor maestro, confidente y amigo que la vida pudo darme, pero aunque su pérdida me duele hasta el fondo de mi corazón estoy feliz por haberlo conocido, por haber sido su alumna, por todos los aprendizajes y risas que nos aventamos, por la confianza que siempre tuvo en mí, por todas esas horas de pláticas, de recuerdos, de música y naftalina.

Gracias Martin porque, aunque ya no estás aquí, sigues enseñándome tanto, dándome tanto. Por abrirme las puertas, levantarme cuando caía, por esmerarte tanto y enseñarme que siempre hay más para dar, a nunca rendirse y jamás perder la humildad.

Te prometo terminar todos los proyectos que planeamos, ir a todas las colecciones que me contabas y a esos lugares que eran tus lugares de paz, para desayunar cuando nada importa más que el momento. A llegar a la cima de las cimas, y hacer brillar aún más tú nombre.

Te extraño mucho, siempre estarás en mi mente y en mi corazón.

\section{AGRADECIMIENTOS}

A David Venegas por la corrección de estilo del manuscrito y a los revisores por sus acertados comentarios y sugerencias al escrito.

Cuadro 1. Producción científica del Dr. Martín L. Zurita-García. Artículos indizados

2006

Mendoza-Ramírez, M., I. Pacheco-Rueda, M. A. Sarmiento-Cordero y M. L. Zurita-García. Entomofilatelia en México: Un tributo a la Amistad de los insectos, Boletín Sociedad Entomológica Aragonesa, 38: 443-449.

2012

Zurita-García, M. L., S. Zaragoza-Cabalero y H. Ochoterena-Booth. New species of the genus Agriotes (Coleoptera: Elateridae) from Mexico with a key to species, Zootaxa, 3568: 53-64.

2013

Ortega, G. y M. L. Zurita-García. Estados ninfales de Edessa reticulata y Edessa jugata (Heteroptera: Pentatomidae: Edessinae) para los estados de Oaxaca y Veracruz, México. Revista Mexicana de Biodiversidad, 84(3): 792-801.

2014

Zurita-García, M. L., P. Johnson y S. ZaragozaCaballero. Biodiversidad de la familia Elateridae (Coleoptera) en México. Revista Mexicana de Biodiversidad, Suplemento 85: 303-311.

2015

Zaragoza Caballero, S. y M. L. Zurita-García. Preliminary study on the phylogeny of the family Phengodidae (Insecta: Coleoptera). Zootaxa, 3497(4): 525-542. 
2016

Martínez Luque, E. O., M. L. Zurita-García y A. Zaldívar-Riverón. Inventario de especies de elatéridos (Coleoptera: Elateridae) de un bosque tropical caducifolio mexicano. Revista Mexicana de Biodiversidad, 87: 956-965.

Villegas, G., E. O. Martínez-Luque y M. L. Zurita-García. Pseudoescorpiones (Arachnida; Pseudoscorpiones) simbiontes de Chalcolepidius approximatus erichson (Coleoptera; Elateridae). Revista Mexicana de Biodiversidad, 87(4): 13691371.

2019

Zaragoza-Caballero, S., G. M. Rodríguez-Mirón., V. Vega-Badillo., M. González-Ramírez., M. L. Zurita-García., D. E. Domínguez-Léon., S. LópezPérez., I. G. Gutiérrez-Carranza., P. Cifuentes-Ruiz., C. X. Pérez-Hernández., E. Ramírez Del Valle y N. Gutiérrez. A checklist of the Coleoptera (Insecta) from Morelos, Mexico. Zootaxa, (1): 001-122.

2020

Zaragoza-Caballero, S., S. López-Pérez., V. VegaBadillo., D. E. Domínguez-León., G. M. RodríguezMirón., M. González-Ramírez., I. G. GutiérrezCarranza., P. Cifuentes-Ruiz y M. L. Zurita-García. Luciérnagas del centro de México (Coleoptera: Lampyridae): descripción de 37 especies nuevas. Revista Mexicana de Biodiversidad, (91) e913104: 1-70.

\section{Artículos arbitrados}

2007

Zurita-García, M. L. y S. Zaragoza-Caballero. La familia Elateridae de la Reserva de la Biosfera "Sierra de Huautla", Morelos, México. Entomología Mexicana, 6(1): 306-308.

2013

Martínez-Luque, E. O. y M. L. Zurita-García. Familia Elateridae (Insecta: Coleoptera) en la estación de biología, Chamela, Jalisco, México. Entomología Mexicana, 12 (2): 1470-1476.

2015

Zurita- García, M. L. y E. O. Martínez-Luque. Estudio preliminar de la familia Elateridae (Coleoptera: Elateroidea) del estado de Guerrero, México, Entomología Mexicana, 2: 816-822.

2016

Zavala-León, E., M. L. Zurita-García, S. ZaragozaCaballero, E. González-Soriano, F. NogueraMartínez y E. Ramírez-García. Distribución temporal de los insectos del suelo en el bosque tropical caducifolio de Santiago Dominguillo, Oaxaca. Entomología Mexicana, 3: 543-548.

2017

Zurita-García, M. L., A. Villasana, M. R. Morales-Garza y F. de la C. Hernández-Hernández. Redescripción e ilustración de la larva y pupa de Chalcolepidius approximatus Erichson, 1841. Revista Investigación MultidisciplinariaUniversidad Simón Bolívar, 15: 77-84.

2018

López-Pérez, S., C. Mayorga-Martínez., S. Zaragoza-Caballero., M. L. Zurita-García., D. E. Domínguez-León., G. M. Rodríguez-Mirón., I. G. Gutiérrez-Carranza., M. González-Ramírez y V. Vega-Badillo. El género Baeus Haliday (Hymenoptera: Scelionidae) y su distribución geográfica en México. Boletín de la Asociación Mexicana de Sistemática de Artrópodos, 2 (2): 1315.

Zurita-García, M. L. y M. Aquino-Romero. Diversidad de los languridos (Coleoptera: Langurinae) de México. Boletín de la Asociación Mexicana de Sistemática de Artrópodos,2 (2): 1112.

2020

Zurita-García, M. L., M. Aquino-Romero., P. Cifuentes-Ruiz., S. López-Pérez., V. Vega-Badillo., M. González-Ramírez., G. M. Rodríguez-Mirón., D. E. Domínguez-León y S. Zaragoza-Caballero. Las avispas Vespa Linnaeus (Vespidae: Vespinae) y su Goliat la Vespa mandarinia Smith. Boletín de la Asociación Mexicana de Sistemática de Artrópodos, 4 (1): 17-19.

2006

Zaragoza Caballero, S., M. A. SarmientoCordero, M. I. Pacheco-Rueda y M. L. ZuritaGarcía. Patrones de distribución de la familia Lycidae, Componentes Bióticos Principales de la Entomofauna Mexicana (pp.562). En: Morrone, J. J. y J. Llorente Bousquets (Eds.). Las Prensas de Ciencias, Vol. 1. Facultad de Ciencias, Universidad Nacional Autónoma de México, México, D. F.

Zaragoza-Caballero, S., F. A. Noguera, E. GonzálezSoriano, E. Ramírez-García y M. L. Zurita-García. Estudio de Estado. Escarabajos de Ixtlahuacán (Elateroidea).(pp. 353-360). En: La Biodiversidad en Colima. CONABIO. México. 
2017

Cifuentes-Ruiz, P., López-Pérez, S. y ZuritaGarcía, M. L. Análisis de secuencias de DNA mitocondrial (CO-I) para la delimitación preliminar de taxones (pp. 326). En: Sistemática Molecular y Bioinformática: guía práctica. Las Prensas Ciencias, Facultad de Ciencias, Universidad Nacional Autónoma de México, México, D. F.

2020

Zaragoza-Caballero, S., M. L. Zurita-García., M. González-Ramírez., D. E. Domínguez-León., I. G. Gutiérrez-Carranza., C. X. Pérez-Hernández., E. Ramírez del Valle., P. Cifuentes-Ruíz., S. LópezPérez., G. M. Rodríguez-Mirón., V. Vega-Badillo y N. Gutiérrez-Trejo (pp 166- 175). Estudio de Estado. Coleoptera (Insecta). En: La Biodiversidad de Morelos. CONABIO, México.

Cuadro 2. Tesis dirigidas $(*)$ y codirigidas $(+)$ por el Dr. Martín Leonel Zurita García.

2017

+Rodríguez González, Stephany Alejandra. Morfología y distribución de Calliphoridae (Diptera) pioneras en la colonización de cadáveres de Sus scrofa (Linnaeus 1758) en México. Facultad de Ciencias, Universidad Nacional Autónoma de México. Licenciatura en Biología.

*Verastegui Oamaña, Yara. Coleópteros de importancia forense en el municipio de Santa María del Tule, Oaxaca, México. Escuela de Ciencias, Universidad Autónoma Benito Juárez, Oaxaca, México. Licenciatura en Biología.

*Zavala León, Erick Antonio. Distribución temporal de Insecta a nivel epigeo en el bosque tropical caducifolio de Santiago Dominguillo, Oaxaca, México. Facultad de Ciencias, Universidad Nacional Autónoma de México. Licenciatura en Biología.

\section{En curso}

+ Acosta Carvajal, Kevin Jack. La familia Elateridae (Insecta: Coleoptera) de la Colección Entomológica de la UAEM. Facultad de Ciencias Biológicas, Universidad Autónoma del Estado de Morelos. Licenciatura en Biología.

* Aquino Romero, Miriam. Sinopsis de la subfamilia Langurinae (Coleoptera: Erotylidae) de México. Facultad de Ciencias, Universidad Nacional Autónoma de México. Licenciatura en Biología.

* Espadín Artigas, Mónica Selene. Coleópteros de

Recibido: 28 octubre 2020

Aceptado: 21 enero 2021 importancia forense de un bosque tropical caducifolio en Aguacatitla, Huasca, Hidalgo. Facultad de Ciencias, Universidad Nacional Autónoma de México. Licenciatura en Biología.

* González Álvarez, Andrea Beatriz. Revisión taxonómica de la tribu Pyrophorini (Familia Elateridae, Coleoptera) de la CNIN. Facultad de Ciencias, Universidad Nacional Autónoma de México. Licenciatura en Biología.

+ Guzmán González, Viridiana. Listado de coleópteros necrófilos de la REPSA, UNAM. Facultad de Medicina, Universidad Nacional Autónoma de México. Licenciatura en Ciencia Forense.

* Juárez Martínez, José Gilberto. Sucesión de coleópteros necrófagos en cerdos domésticos en el municipio de San Agustín Loxicha, Pochutla, Oaxaca, México. Sistemas Biológicos e Innovación Tecnológica, Universidad Autónoma Benito Juárez, Oaxaca, México. Licenciatura en Biología.

+ Martínez Gálvez, Ángel de Jesús. Listado de especies de Elateridae (Coleoptera: Elateridae) del estado de Morelos. Facultad de Ciencias Biológicas, Universidad Autónoma del Estado de Morelos. Licenciatura en Biología.

Cuadro 3. Participaciones del Dr. Martín Leonel Zurita García en exámenes de grado.

2013

López Pérez, Sara. Revisión taxonómica e inferencia del género Ogdoecosta Spaeth, 1909 (Coleoptera: Chrysomelidae: Cassidinae). Instituto de Biología, Universidad Nacional Autónoma de México. Maestría en Ciencias Biológicas.

\section{4}

García Rivera, Griselda. Diversidad de escarabajos acuáticos atraídos a trampas de luz en selva baja caducifolia de la vertiente del Pacífico Mexicano. Instituto de Biología, Universidad Nacional Autónoma de México. Maestría en Ciencias Biológicas.

\section{8}

Martínez Luque, Erick Omar. Distribución conocida y potencial del género Scaptolenus LeConte (Elateridae: Elaterinae: Cebrionini). Facultad de Ciencias Naturales, Universidad Autónoma de Querétaro. Maestría en Ciencias Biológicas.

Nestor Arreola, Jorge Ismael. Sistemática de las especies de Brachiacantha Dejean, 1837 (Coleoptera: Coccinellidae) pertenecientes al grupo Dentipes. Universidad Autónoma de Morelos. Doctorado en Ciencias Naturales. 

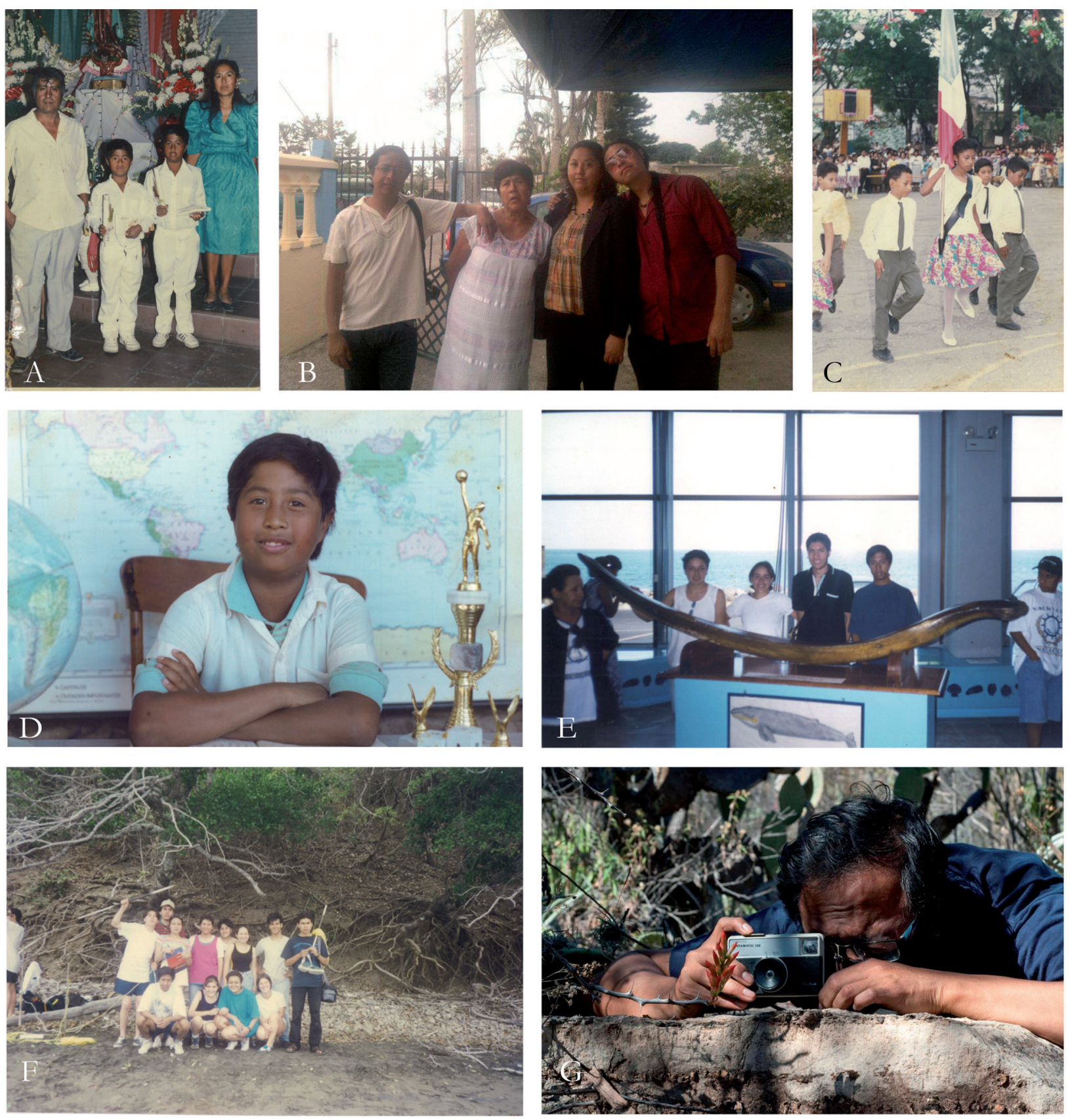

Figura 1. A) Primera comunión de Martín a lado de su papá, mamá y hermano (Deynis). B) Martín junto a su mamá, hermana y hermano (María Areyni y Kaedi). C) Martín como parte de la escolta en su escuela primaria. D) Retrato de Martín en la primaria. E-F) Prácticas de campo. G) Martín fotografiando la naturaleza. 

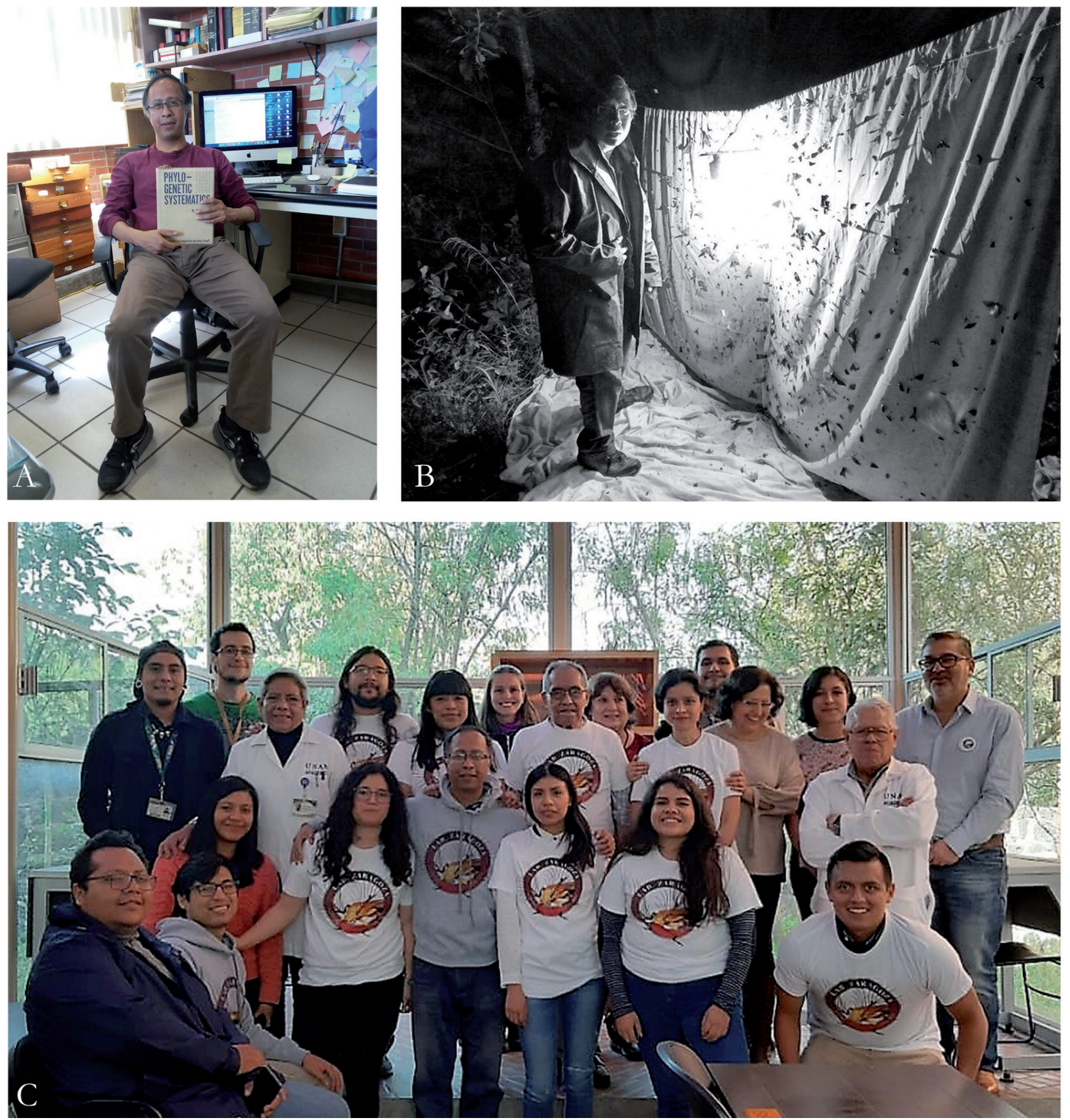

Figura 2. A) Martín en el cubículo del Dr. Santiago Zaragoza. B) Colecta de campo nocturna. C) Celebración de fin de año (2019) junto al Dr. Santiago Zaragoza, parte de su laboratorio y compañeros de la Colección Nacional de Insectos. 


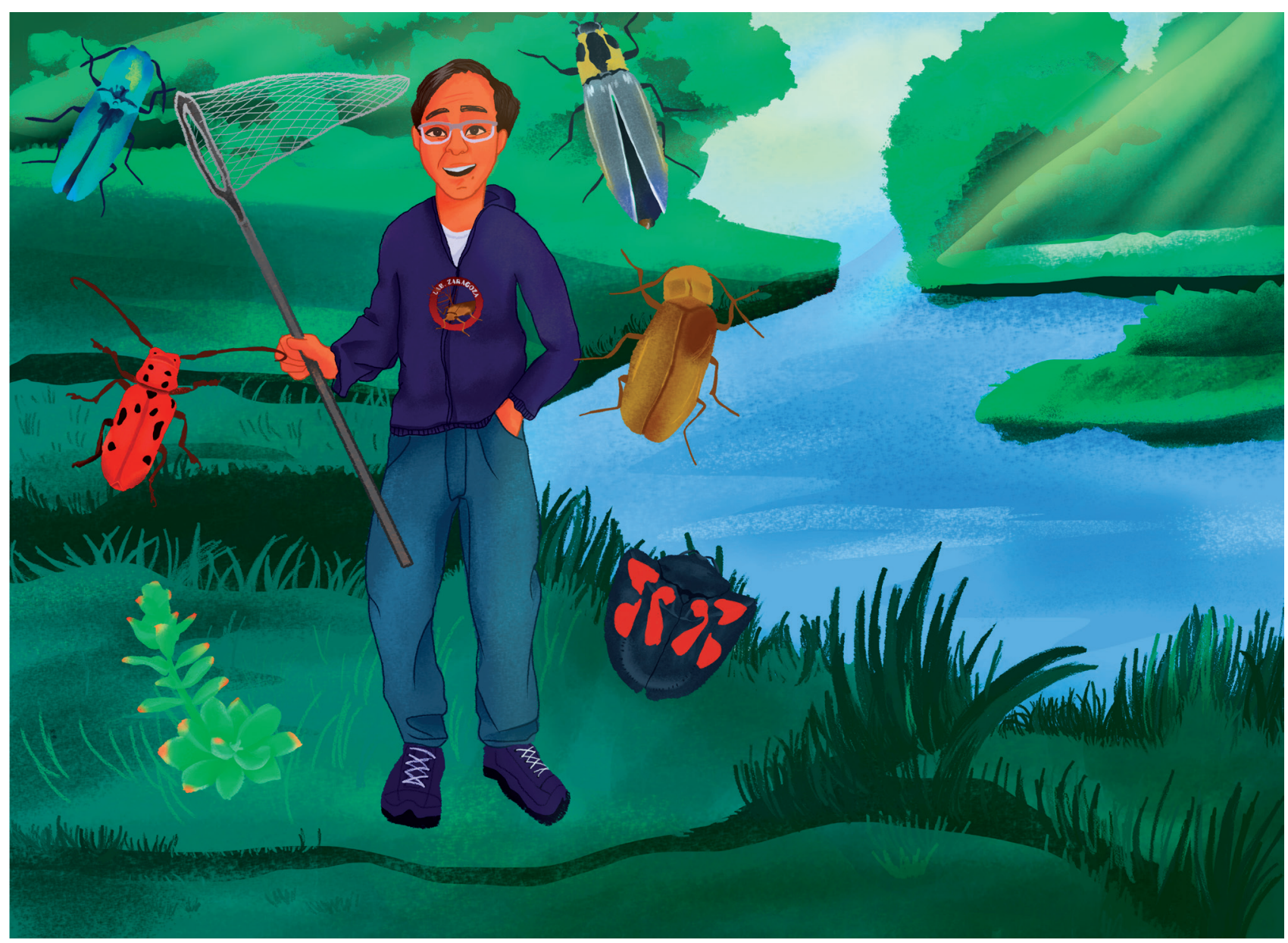

Figura 3. Ilustración en homenaje a Martín L. Zurita García. 